\title{
Copy number changes of CRISP3 in oral squamous cell carcinoma
}

\author{
WEN-CHANG KO ${ }^{1,2,3}$, KEISUKE SUGAHARA ${ }^{1}$, TAKUMI SAKUMA ${ }^{1}$, CHING-YU YEN ${ }^{2,3}$, \\ SHYUN-YEU LIU ${ }^{2,3}$, GWO-AN LIAW ${ }^{2}$ and TAKAHIKO SHIBAHARA ${ }^{1}$ \\ ${ }^{1}$ Department of Oral and Maxillofacial Surgery, Tokyo Dental College, Mihama, Chiba 261-8502, Japan; \\ ${ }^{2}$ Department of Oral and Maxillofacial Surgery, Chi-Mei Medical Center, Yongkang; \\ ${ }^{3}$ School of Dentistry, Taipei Medical University, Taipei, Taiwan, R.O.C.
}

Received April 13, 2011; Accepted August 23, 2011

DOI: $10.3892 / \mathrm{ol} .2011 .418$

\begin{abstract}
The aim of this study was to identify tumor suppressor genes (TSGs) in oral squamous cell carcinoma (OSCC) using whole-genome analysis of microarray technology and real-time quantitative polymerase chain reaction (QPCR). We applied whole-genome analysis of TSGs in the specimens from 3 patients of OSCC by microarray technology. A total of 11 genes, CRISP3, SCGB3A1, AGR2, PIP, C20orf114, TFF1, STATH, AZGP1, MUC7, DMBT1 and $L O C 389429$, were found to be down-regulated, and 2, matrix metallopeptidase (MMP) 1 and $M M P 3$, were found to be up-regulated in the 3 OSCC patients using microarray technology. In this study, we selected the CRISP3 gene. CRISP3 belongs to the cystein-rich secretary protein gene family in chromosome 6p12.3. CRISP3 has been found in the salivary gland, spleen and prostate gland and is a prominent biomarker in the gene expression of prostate cancer. Down-regulation of this gene was previously observed in OSCC. No studies examining the DNA copy number of CRISP3 in detail exist. We analyzed the DNA copy number of CRISP3 in 5 OSCCderived cell lines (SAS, Ca9-22, KON, HSC2 and HSC4) and 60 OSCC tissues by real-time QPCR. The DNA copy number loss of CRISP3 was observed in 2 of the 5 OSCC-derived cell lines (SAS, HSC2) and in 24 of 60 patients (40.0\%) using real-time QPCR. A significant statistical correlation between the copy number loss and gender and $\mathrm{T}$ classification was observed. These results indicate that the inactivation of CRISP3 is an early event in OSCC, since the T1/T2 classification is correlated with DNA copy number loss of CRISP3, whereas T3/T4 classification is not. We conclude that CRISP3 may be involved in the carcinogenesis of OSCC.
\end{abstract}

Correspondence to: Dr Takahiko Shibahara, Department of Oral and Maxillofacial Surgery, Tokyo Dental College, Mihama, Chiba 261-8502, Japan

E-mail: sibahara@tdc.ac.jp

Key words: oral squamous cell carcinoma, tumor suppressor gene, microarray technology, real-time quantitative polymerase chain reaction, DNA copy number

\section{Introduction}

Oral squamous cell carcinoma (OSCC) is a common malignancy that affects 300,000 individuals per year worldwide (1). A number of etiologic factors have been implicated in the development of OSCCs, such as the use of tobacco, alcohol or betel nut chewing, human papillomavirus infection, and the presence of incompatible prosthetic materials. Tobacco is a major risk factor for the development of OSCC and overall risk of OSCC among smokers is 7-10 times higher than that of non-smokers (2-4).

The primary treatment for management of OSCC is surgical intervention. Despite considerable advances in the treatment of OSCC over the past two decades, overall disease outcomes have only modestly improved (5). Local tumor recurrence affects approximately $60 \%$ of patients and metastasis develops in $15-25 \%$ (6). The prevention and management of this disease is likely to greatly benefit from the identification of molecular markers and targets $(7,8)$.

However, little is known about the molecular basis of OSCC compared with other malignancies. Molecular alterations in a number of oncogenes and tumor suppressor genes (TSGs) associated with the development of OSCC may be significant clues with which to address these problems $(9,10)$.

Inactivation of TSG is considered to be associated with carcinogenesis, and alterations in TSGs are accepted to be critical events in the multi-step process leading toward the development of cancer. Loss of chromosome 3p22-24 is a common early genetic event in OSCC (11). Specific regions of arm harbor candidate tumor suppressor genes including FHIT and RSSFIA. It is generally accepted that the transformation of normal tissue into malignant tissue follows an accumulation of genetic changes in the TSGs and oncogenes (9). High throughput investigation into the molecular characteristics of OSCC has mainly utilized microarray technology to search for gene expression profiles associated with disease and disease outcome.

In the present study, microarray technology was applied to screen novel TSGs in OSCC patients, and we selected candidate gene CRISP3. CRISP3 DNA copy number was evaluated by real-time QPCR in 5 OSCC-derived cell lines and 60 primary OSCC samples. Human CRISP3 is also likely to be involved in the pathogenesis of prostate cancer, where CRISP3 expression is significantly upregulated (12). 
CRISP3 is secreted and can be detected in human tissue fluids including saliva, sweat, blood and seminal plasma, rendering it an ideal candidate biomarker for pathophysiological conditions, including ectopic pregnancy (12-14). Ye et al reported the expression of CRISP3 in OSCC using microarray technology (15). Therefore, the purpose of the present study was to assess the role of CRISP3 in OSCC.

\section{Materials and methods}

Cells. The 5 human OSCC-derived cell lines used in this study were SAS, Ca9-22, KON, HSC2, and HSC4 (Human Science Research Resources Bank, Osaka, Japan). SAS was from male tongue, Ca9-22 from male gingiva, KON from male oral floor, HSC2 from male mouth and HSC4 from male tongue. The cell lines were maintained at $37^{\circ} \mathrm{C}$ (humidified atmosphere 5\% CO $2 / 95 \%$ air) in $150 \times 200 \mathrm{~mm}$ tissue culture dishes (Nunc, Roskilde, Denmark) and cultured in Dulbecco's modified Eagle's medium F-12 HAM (Sigma, St. Louis, MO, USA) with $10 \%$ fetal bovine serum (Sigma) plus $50 \mathrm{U} / \mathrm{ml}$ penicillin and streptomycin.

Normal oral keratinocyte (NOKs) strains from two patients who had undergone dental surgery served as the controls, and the patients provided written informed consent prior to the start of the study. The normal oral specimens were washed in Dulbecco's phosphate-buffered saline (PBS) (Sigma) and then placed overnight in $0.25 \%$ trypsin-EDTA solution (Sigma) at $4^{\circ} \mathrm{C}$. After the epithelial tissue was separated from the connective tissue, it was disaggregated by incubation in $0.25 \%$ trypsin-EDTA solution for $15 \mathrm{~min}$ with gentle pipetting at $37^{\circ} \mathrm{C}$. Isolated epithelial cells were then seeded into Collagen I Cellware 60-mm dish biocoat cell environments (Becton Dickinson Labware, Bedford, MA, USA) and cultured in Keratinocyte Basal Medium-2 (Cambrex, Walkersville, MD, USA) with $0.4 \%$ bovine pituitary extract, $0.1 \%$ human epidermal growth factor, $0.1 \%$ insulin, $0.1 \%$ hydrocortisone, $0.1 \%$ transferrin, $0.1 \%$ epinephrine and $0.1 \%$ GA-1000 (Cambrex) (16).

Patient characteristics. A total of 60 patients with OSCC were included in the present study. Surgical resection of primary tumors and marginal normal tissues from all patients was performed at the Hospital of Chimei, Tainan, Taiwan, and the Hospital of Tokyo Dental College, Chiba, Japan, between July 1999 and September 2009. Written informed consent was obtained from all patients and the study approved by the ethics committees of the Hospital of Chimei and the Tokyo Dental College, ethical clearance number 205. Informed consent was obtained from each patient prior to surgical resection.

Staging of tumors was performed according to the International Union Against Cancer TNM staging system (17). Cervical LNM during the 12-month follow-up period was evaluated by computed tomography and magnetic resonance imaging. In case of a positive signal, metastasis was further confirmed by histopathological examination of the resected tissues. Patients not exhibiting any cervical LNM for 12 months after surgery were considered metastasisfree. Patients with distant metastasis at the time of clinical examination or those receiving preventive radiotherapy or chemotherapy were excluded from this study. Detailed patient characteristics are shown in Table I.

Primary tumor samples. Resected primary tumor tissues were divided into two sections. Of these, one section was frozen immediately in liquid nitrogen and stored at $-80^{\circ} \mathrm{C}$. The other was fixed in $10 \%$ formalin for histopathological examination. The resected marginal normal tissues were frozen immediately in liquid nitrogen and stored at $-80^{\circ} \mathrm{C}$.

Microarray gene expression profiling. A total of 3 OSCC patients ( 2 tongue and 1 oral floor patient) were subjected to whole-genome analysis using microarray technology to determine the TSGs.

Total RNA was extracted from 3 OSCC patients using the Qiagen RNeasy mini kit and the SuperScript double-stranded cDNA synthesis kit (Invitrogen) was used to generate cDNA according to the manufacturer's instructions. Cy3 labeling of ds-cDNA was performed overnight using the NimbleGen One-Color DNA labeling kit.

Cy3-labeled ds-cDNA $(4 \mu \mathrm{g})$ was hybridized to the Homo sapiens $4 \times 72 \mathrm{~K}$ gene expression array (Roche NimbleGen) representing 24,000 protein-coding genes, according to the manufacturer's instructions. The mRNA expression data were analyzed using NimbleScan software version 2.4, which applied quintile normalization (18), and expression values were obtained using the Robust MultiChip Average algorithm as described by Irizarry et al (19). Expressional alterations of 2-fold across the two biological repeats were considered significant.

DNA copy number analysis by real-time QPCR. DNA from the frozen 60 tumor and marginal normal tissues was extracted using the QIAamp tissue kit (Qiagen, Hilden, Germany) according to the manufacturer's instructions.

Real-time QPCR was performed using 60 tumor and marginal normal tissue samples with SYBR-Green I fluorescence detection on a LightCycler (Roche Diagnostics, Basel, Switzerland). Oligonucleotide primers for real-time QPCR were designed using Primer 3 software (Whitehead Institute for Biomedical Research), and uniqueness in the human genome was confirmed using a BLAST search. The primer set specific for CRISP3 was forward: 5'-ATCAGGCTGCATC CCAATAC-3', and reverse: 5'-AACACCAAATCCCCACA GAA-3'. The 20- $\mu 1$ reaction mixture consisted of $10 \mu \mathrm{l} 2 \mathrm{X}$ iQ SYBR-Green Supermix (Bio-Rad Laboratories, Hercules, CA, USA), $10 \mathrm{ng}$ genomic DNA, and $800 \mathrm{nM}$ of each PCR primer. The reaction mixture was loaded into glass capillary tubes and submitted to an initial denaturation at $95^{\circ} \mathrm{C}$ for $10 \mathrm{~min}$, followed by 45 cycles of amplification at $95^{\circ} \mathrm{C}$ for $10 \mathrm{sec}$ for denaturation, $58^{\circ} \mathrm{C}$ for $10 \mathrm{sec}$ for annealing, and $72^{\circ} \mathrm{C}$ for $15 \mathrm{sec}$ for extension, with a temperature slope of $20^{\circ} \mathrm{C} / \mathrm{sec}$, performed in the LightCycler. The crossing point for each amplification curve was determined by the second derivative maximum method. The copy numbers are presented as the log ratio of each target locus in tumor normalization to internal reference loci $(G A P D H)$ and relative to the normal DNA. The primer set specific to $G A P D H$ was forward: 5'-CCACTAGGCGCTCACTGTTCT-3', and reverse: 5'-GCG AACTCACCCGTTGACT-3'. DNA copy number loss was 
Table I. Clinical characteristics of 60 OSCC patients.

\begin{tabular}{|c|c|c|c|c|c|c|c|c|c|c|}
\hline Case & Gender & Age & Ethnic group & Tumor site & $\mathrm{T}$ & $\mathrm{N}$ & Stage & $\mathrm{pN}$ & Tobacco & Alcohol \\
\hline 1 & M & 43 & Taiwanese & Buccal mucosa & 3 & 0 & III & - & + & + \\
\hline 2 & M & 66 & Taiwanese & Lower gingiva & 2 & 0 & II & - & + & + \\
\hline 3 & M & 31 & Taiwanese & Tongue & 2 & 0 & II & - & + & + \\
\hline 4 & $\mathrm{M}$ & 55 & Taiwanese & Buccal mucosa & 1 & 0 & $\mathrm{I}$ & - & + & + \\
\hline 5 & M & 37 & Taiwanese & Tongue & 3 & 0 & III & - & + & + \\
\hline 6 & M & 58 & Taiwanese & Buccal mucosa & 1 & 0 & $\mathrm{I}$ & - & + & + \\
\hline 7 & $\mathrm{~F}$ & 71 & Taiwanese & Upper gingiva & 2 & 0 & II & - & - & - \\
\hline 8 & M & 43 & Taiwanese & Oral floor & 2 & 0 & II & - & + & + \\
\hline 9 & M & 39 & Taiwanese & Buccal mucosa & 2 & 0 & II & - & + & + \\
\hline 10 & $\mathrm{M}$ & 56 & Taiwanese & Tongue & 1 & 0 & $\mathrm{I}$ & - & + & + \\
\hline 11 & M & 49 & Taiwanese & Buccal mucosa & 3 & 0 & III & - & + & + \\
\hline 12 & M & 72 & Taiwanese & Tongue & 1 & 1 & III & + & + & + \\
\hline 13 & $\mathrm{M}$ & 51 & Taiwanese & Lower gingiva & 4 & 0 & IV & - & + & + \\
\hline 14 & M & 55 & Taiwanese & Tongue & 2 & 2 & IV & + & - & - \\
\hline 15 & M & 59 & Taiwanese & Buccal mucosa & 3 & 1 & III & + & - & - \\
\hline 16 & M & 54 & Taiwanese & Buccal mucosa & 1 & 0 & I & - & + & + \\
\hline 17 & M & 68 & Taiwanese & Buccal mucosa & 2 & 0 & II & - & + & - \\
\hline 18 & M & 33 & Japanese & Tongue & 2 & 1 & III & + & - & + \\
\hline 19 & M & 55 & Japanese & Oral floor & 3 & $2 c$ & IV & + & + & + \\
\hline 20 & M & 74 & Japanese & Tongue & 2 & 1 & III & - & + & + \\
\hline 21 & $\mathrm{M}$ & 54 & Japanese & Buccal mucosa & 4 & $2 c$ & IV & + & - & + \\
\hline 22 & M & 64 & Japanese & Lower gingiva & 4 & $2 \mathrm{a}$ & IV & + & - & + \\
\hline 23 & M & 53 & Japanese & Tongue & 2 & 1 & III & + & + & - \\
\hline 24 & $\mathrm{~F}$ & 70 & Japanese & Buccal mucosa & 3 & 1 & III & + & - & - \\
\hline 25 & M & 44 & Japanese & Tongue & 1 & 0 & $\mathrm{I}$ & - & - & + \\
\hline 26 & $\mathrm{~F}$ & 50 & Japanese & Upper gingiva & 1 & 0 & $\mathrm{I}$ & - & - & + \\
\hline 27 & M & 36 & Japanese & Upper gingiva & 2 & 0 & II & - & - & + \\
\hline 28 & $\mathrm{~F}$ & 37 & Japanese & Tongue & 2 & $2 c$ & IV & - & - & - \\
\hline 29 & $\mathrm{M}$ & 47 & Japanese & Tongue & 2 & 0 & II & + & - & + \\
\hline 30 & M & 43 & Japanese & Tongue & 1 & 0 & $\mathrm{I}$ & - & - & + \\
\hline 31 & M & 57 & Japanese & Tongue & 2 & $2 b$ & IV & - & + & + \\
\hline 32 & M & 52 & Japanese & Tongue & 1 & 0 & I & - & + & + \\
\hline 33 & $\mathrm{~F}$ & 58 & Japanese & Tongue & 2 & 0 & II & - & - & - \\
\hline 34 & M & 59 & Japanese & Upper gingiva & 3 & 1 & III & - & - & + \\
\hline 35 & M & 62 & Japanese & Tongue & 1 & 0 & I & - & + & + \\
\hline 36 & $\mathrm{~F}$ & 70 & Japanese & Upper gingiva & 2 & 1 & III & - & - & - \\
\hline 37 & M & 82 & Japanese & Upper gingiva & 3 & 0 & III & - & - & + \\
\hline 38 & $\mathrm{~F}$ & 69 & Japanese & Buccal mucosa & 2 & 0 & II & - & - & - \\
\hline 39 & M & 80 & Japanese & Lower gingiva & 2 & $2 b$ & IV & + & - & - \\
\hline 40 & M & 48 & Japanese & Tongue & 1 & 0 & I & - & - & + \\
\hline 41 & M & 70 & Japanese & Lower gingiva & 2 & 1 & III & - & + & + \\
\hline 42 & M & 49 & Japanese & Lower gingiva & 2 & $2 b$ & IV & - & - & - \\
\hline 43 & M & 85 & Japanese & Tongue & 2 & 1 & III & - & - & - \\
\hline 44 & M & 71 & Japanese & Lower gingiva & 4 & 1 & IV & - & - & + \\
\hline 45 & $\mathrm{~F}$ & 73 & Japanese & Tongue & 1 & 0 & I & - & - & - \\
\hline 46 & $\mathrm{~F}$ & 58 & Japanese & Lower gingiva & 3 & 0 & III & - & - & - \\
\hline 47 & M & 41 & Japanese & Tongue & 3 & $2 b$ & IV & - & + & + \\
\hline 48 & M & 58 & Japanese & Tongue & 1 & 1 & III & + & + & + \\
\hline 49 & $\mathrm{~F}$ & 66 & Japanese & Buccal mucosa & 2 & 1 & III & + & - & - \\
\hline 50 & M & 63 & Japanese & Tongue & 1 & 0 & I & - & + & + \\
\hline 51 & $\mathrm{~F}$ & 60 & Japanese & Lower gingiva & 2 & 0 & II & - & - & - \\
\hline 52 & M & 52 & Japanese & Tongue & 2 & 0 & II & - & + & + \\
\hline 53 & $\mathrm{~F}$ & 72 & Japanese & Upper gingiva & 2 & $2 \mathrm{a}$ & IV & - & - & - \\
\hline 54 & $\mathrm{~F}$ & 63 & Japanese & Lower gingiva & 4 & $2 b$ & IV & - & - & - \\
\hline 55 & $\mathrm{~F}$ & 66 & Japanese & Buccal mucosa & 1 & 0 & I & - & - & - \\
\hline 56 & M & 66 & Japanese & Oral floor & 4 & $2 c$ & IV & + & + & + \\
\hline 57 & M & 66 & Japanese & Lower gingiva & 4 & $2 b$ & IV & - & - & + \\
\hline 58 & M & 76 & Japanese & Oral floor & 2 & 1 & III & + & - & + \\
\hline 59 & M & 77 & Japanese & Oral floor & 2 & 1 & III & - & - & + \\
\hline 60 & $\mathrm{~F}$ & 69 & Japanese & Lower gingiva & 4 & $2 b$ & IV & + & - & - \\
\hline
\end{tabular}


Table II. List of aberrantly expressed genes.

$$
\begin{array}{lll}
\text { Gene symbol Gene name Location } & \text { Le }
\end{array}
$$

Upregulated genes

$M M P 1$

$M M P 3$

Downregulated genes
Matrix metallopeptidase 1

$11 \mathrm{q} 22$

Matrix metallopeptidase 3

$11 \mathrm{q} 22$

STATH
MUC7
SCGB3A1
CRISP3
LOC389429
AGR2
AZGP1
PIP
DMBT1
C20orf114
TFF1

determined as $<1.2$ (20). The data were analyzed as the mean $\pm \mathrm{SD}$ of three independent experiments with samples in triplicate.

Statistical analysis. The association between copy number loss and any clinical findings were assessed by the Fisher's exact test. The association between the DNA copy number of tumor and normal tissues was assessed by the unpaired $\mathrm{U}$ test (SAS Institute, Cary, NC, USA). P- $<0.05$ was considered to indicate statistical significance.

\section{Results}

Clinicopathological findings. This study included 60 patients who had undergone surgical resection of primary OSCC. The patients, 45 males and 15 females, had average ages of 56.7 years for the males (range 31-85) and 63.1 years for the females (range 37-73). Regarding patient ethnicity,17 patients of Taiwanese and 43 of Japanese ethnicity were included in this study. The tumor sites were as follows: 23 patients, tongue; 13, buccal mucosa; 12, lower gingiva; 7, upper gingiva; and 5, oral floor. The T classifications, which indicate the sizes of the primary clinical tumors, were as follows: 15 patients with $\mathrm{T} 1$, 27 with T2, 10 with T3 and 8 with T4. The classifications by TNM stage were: 13 patients with Stage I, 12 with Stage II, 19 with Stage III, and 16 with Stage IV. A total of 18 of the 60 patients had histopathologically-confirmed cervical lymph node metastasis (LNM) at the time of diagnosis or during the 12-month follow-up period (LNM present).

Microarray analysis. Three OSCC patients were subjected to microarray analysis to screen for TSGs in this population. As in previous studies, we know that there are 17 upregulated genes in OSCC, comprising matrix metallopeptidase 1 MMP1, MMP10, MMP3, MMP12, PTHLH, INHBA, LAMC2, IL8, KRT17, COL1A2, IF16, ISG15, PLAU, GREM1, MMP9, IFI44 and CXCLI, and that there are 18 downregulated genes in OSCC, comprising KRT4, MAL, CRNN, SCEL, CRISP3, SPINK5, CLLA4, ADH1B, P11, TGM3, RHCG, PPP1R3C, CEACAM7, HPGD, CFD, ABCA8, CLU and CYP3A5 $(15,21)$. In this study, we found 2 upregulated genes, $M M P 1$ and $M M P 3$, and 11 downregulated genes, CRISP3, SCGB3A1, AGR2, PIP, C20orf114, TFF1, STATH, AZGP1, MUC7, $D M B T 1$ and LOC389429, in all three patients. The detected genes of up- and down-regulation are shown in Table II. CRISP3 was selected as it is currently unknown as to whether this gene is associated with OSCC.

Assessment of CRISP3 by real-time QPCR. To evaluate microarray data at CRISP3, real-time QPCR was performed using non-amplified genomic DNA as a template. Initially, PCR primer sets located in CRISP3 were successfully designed to meet the criteria for reliable quantification. One PCR primer set was designed in a housekeeping gene, GAPDH, located on chromosome $12 \mathrm{p}$, as a reference for normalization. Real-time QPCR analysis was performed with the above-mentioned primer sets using genomic DNA of 5 OSCC cell lines and 60 OSCC patients. DNA copy number loss of CRISP3 was observed in 2 of the 5 OSCC-derived cell lines (SAS and HSC2). The copy number of CRISP3 was significantly reduced in OSCC-derived cell lines compared with NOKs $(\mathrm{P}=0.024$, Unpaired U-test: Fig. 1). The copy number loss of CRISP3 was observed in $24(40 \%)$ of the 60 patient specimens. We evaluated statistically significant differences in all clinical characteristics pertaining to the CRISP3 copy number between OSCCs and normal tissues. No statistically significant differences were noted. Moreover, no statistically significant differences were observed in the CRISP3 copy number between OSCC tumor tissues and normal tissues in the 60 OSCC patients $(\mathrm{P}=0.401$, Mann-Whitney's U-test; Fig. 2).

Clinicopathological findings and statistical analysis. We compared our results with the clinicopathological findings for each tumor. The copy number loss of CRISP3 was observed 


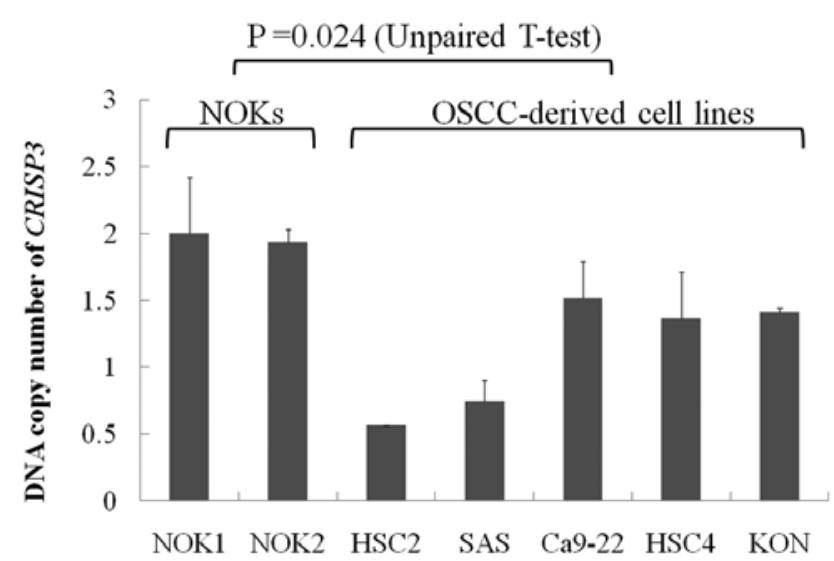

Figure 1. Quantification of CRISP3 DNA copy numbers in OSCC-derived cell lines and NOKs by real-time QPCR. Two OSCC cell lines decreased CRISP3 copy numbers compared with NOKs. A statistically significant difference was found in the CRISP 3 copy numbers between OSCC-derived cell lines and NOKs $(\mathrm{P}=0.024$, unpaired $\mathrm{U}$ test $)$.

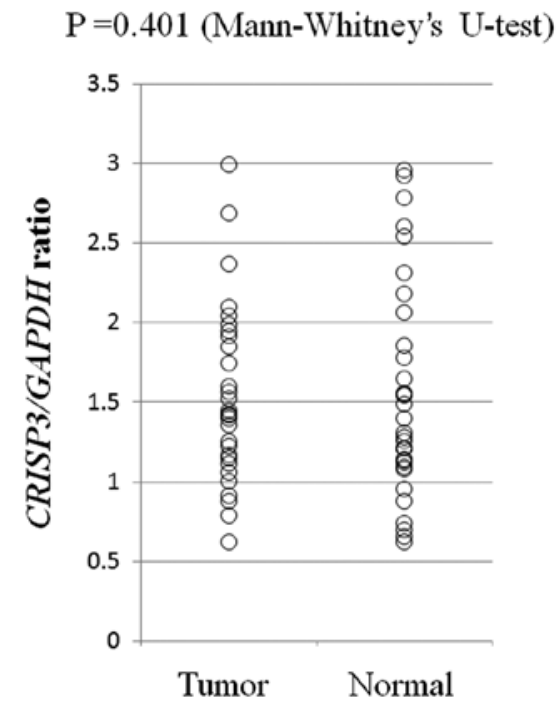

Figure 2. DNA copy numbers of CRISP 3 in 60 OSCC patients. The manifestation amount of DNA copy numbers of CRISP3 among tumor and normal tissues. The statistically significant difference could not be confirmed.

in $24(40 \%)$ of the 60 patients. The Fisher's exact test was performed to evaluate the significance of correlations between copy number loss of CRISP3 and clinicopathological findings (Table III). A significant statistical correlation between the copy number loss and gender and $\mathrm{T}$ classification was observed. The copy number loss of CRISP3 was detected in early stage lesions; and the copy number loss of CRISP3 tended to be higher in early clinical stages. Moreover, no statistically significant correlation was found between the copy number loss of CRISP3 and other clinicopathological findings such as age, ethnicity, lymph node metastasis, tobacco and alcohol associated with the tumor samples.

\section{Discussion}

OSCC is often associated with loss of eating and speech function, disfigurement and psychological distress. The
Table III. Correlation between the DNA copy numbers of CRISP3 and clinical characteristics in OSCCs.

\begin{tabular}{|c|c|c|c|}
\hline & Loss & Normal & P-value \\
\hline \multicolumn{4}{|l|}{ Gender } \\
\hline Male & 13 & 32 & \multirow[t]{2}{*}{0.005} \\
\hline Female & 11 & 4 & \\
\hline \multicolumn{4}{|l|}{ Age } \\
\hline$<65$ & 17 & 21 & \multirow[t]{2}{*}{0.104} \\
\hline$\geq 65$ & 15 & 7 & \\
\hline \multicolumn{4}{|l|}{ Ethnic group } \\
\hline Taiwanese & 6 & 11 & \multirow[t]{2}{*}{0.773} \\
\hline Japanese & 18 & 25 & \\
\hline \multicolumn{4}{|l|}{ Tumor site } \\
\hline Tongue & 6 & 17 & \multirow[t]{5}{*}{0.491} \\
\hline Buccal mucosa & 6 & 7 & \\
\hline Lower gingiva & 6 & 6 & \\
\hline Upper gingiva & 4 & 3 & \\
\hline Oral floor & 2 & 3 & \\
\hline \multicolumn{4}{|l|}{$\mathrm{T}$ classification } \\
\hline $\mathrm{T} 1 / \mathrm{T} 2$ & 21 & 21 & \multirow[t]{2}{*}{0.021} \\
\hline $\mathrm{T} 3 / \mathrm{T} 4$ & 3 & 15 & \\
\hline \multicolumn{4}{|l|}{ LNM } \\
\hline Present & 6 & 12 & \multirow[t]{2}{*}{0.573} \\
\hline Absent & 18 & 24 & \\
\hline \multicolumn{4}{|l|}{ Stage } \\
\hline I & 5 & 8 & \multirow[t]{4}{*}{0.111} \\
\hline II & 8 & 4 & \\
\hline III & 6 & 13 & \\
\hline IV & 5 & 11 & \\
\hline \multicolumn{4}{|l|}{ Tobacco } \\
\hline Yes & 7 & 19 & \multirow[t]{2}{*}{0.11} \\
\hline No & 17 & 17 & \\
\hline \multicolumn{4}{|l|}{ Alcohol } \\
\hline Yes & 12 & 27 & \multirow[t]{2}{*}{0.058} \\
\hline No & 12 & 9 & \\
\hline
\end{tabular}

LNM, lymph node metastasis confirmed by histopathological examination within the 12-month follow-up period.

development of OSCC is strongly associated with smoking and excessive alcohol consumption (22). The prevention and management of this disease is likely to benefit from the identification of molecular markers and targets $(7,8)$.

Recently, the development of tools for measuring gene expression and copy numbers across the entire genome has revolutionized our ability to characterize cancers at the molecular level. Cytogenetic analyses in conjunction with molecular genetics analyses by a number of research groups have shown an accumulation of genetic abnormalities during the development and/or progression of OSCC (23). In this study, we identified 11 downregulated genes, CRISP3, SCGB3A1, AGR2, PIP, C20orf114, TFF1, STATH, AZGP1, $M U C 7, D M B T 1$ and LOC389429, and 2 up-regulation genes, 
MMP1 and MMP3, using microarray technology. CRISP3 was selected due to the fact that it is unknown as to whether CRISP3 is associated with OSCC. However, to the best of our knowledge no previous OSCC studies have identified CRISP3 as we observed in the current study. Our findings indicate that DNA copy number loss of CRISP3 is involved in carcinogenesis in OSCC patients.

Little is known about the function of the mammalian CRISPs; however, CRISP1 and CRISP2 are known to be involved in various steps in reproduction (24). The C-terminal domain of CRISP2 has been shown to interact with calcium channels (25) and to bind a kinase present in the acrosome of mouse sperm (26). Less is known about human CRISP3. CRISP3, which is also known as specific granule protein of $28 \mathrm{kDa}$ (SGP28), belongs to a family of CRISPs characterized by their size (220-230 amino acids), their secretory properties and a content of 16 highly conserved cysteine residues, which form an intra-molecular disulphide bond (27). Apart from its ability to bind A1BG in serum, Udby et al have shown that CRISP3 forms similar complexes with one of the three major proteins secreted from the prostate in seminal plasma, $\beta$-microseminoprotein (28). Human CRISP3 is also likely to be involved in the pathogenesis of prostate cancer, where CRISP3 expression is significantly upregulated (12). However, to the best of our knowledge no previous reports have identified the CRISP3 gene in OSCC.

Table II lists other candidate genes that may be associated with carcinogenesis in OSCC. These genes exhibit correlations with various types of carcinoma excluding C20orf114, STATH, and LOC389429. SCGB3Al (HINI) is a TSG that is highly expressed in a number of epithelial tissues, including the breast, lung, trachea, pancreas, prostrate and salivary gland. Inactivation of SCGB3A1 expression by promoter methylation is frequent in many types of epithelial carcinoma and carcinoma in situ, including breast, lung and nasopharyngeal carcinoma $(29,30)$. AGR2 is a putative member of the protein disulfide isomerase family and was first identified as a homolog of the Xenopus laevis gene XAG-2. AGR2 was downregulated in gastric tumor tissue compared to the control (31). AGR2 has previously been found to be one of several genes that encode secreted proteins showing an increased expression in prostate cancer cells compared to normal prostatic epithelium (32). We observed that the MMPl gene is up-regulated in OSCC. MMPI is located on chromosome 11q22.3 and belongs to the MMP family, which is responsible for the degradation of extracellular matrix components. There is clear evidence indicating that $M M P 1$ is involved in various cell and tumour events, including cancer-cell development, growth, proliferation, apoptosis, invasion and metastasis, as well as angiogenesis and immune surveillance (33-36).

Our results suggest that the CRISP3 gene is a novel TSG particular to OSCC, and inactivation of the CRISP3 gene may play one or more roles in the carcinogenesis of OSCCs. In the present study, we applied whole-genome analysis of TSGs in specimens from 3 patients with OSCC by microarray techno-logy. A significant statistical correlation was observed between the DNA copy number of CRISP3 and T-classification and gender. No significant correlation was found between DNA copy number loss of CRISP3 and age, ethnic group, tumor site, lymph node metastasis, tumor stage, tobacco or alcohol. In this population, 15 patients were habitual consumers of tobacco, alcohol and/or betel nuts. We compared these 15 patients with 22 patients who did not consume tobacco, alcohol or betel nuts. No significant correlation was found between the DNA copy number loss of CRISP3 and tobacco, alcohol and/or betel nut consumption. These results indicate that inactivation of the CRISP3 is an early event in OSCC, since T1/T2 classification is correlated with DNA copy number loss of CRISP3 rather than T3/ T4 classification. We were not able to apply the functional analysis of the CRISP3 gene. Further studies employing techniques including immunoblotting, immunofluorescence, and immunohistochemistry are required to clarify the function of this gene in the development and progression of OSCC. Furthermore, a study with a larger patient series is requred to validate these results, in order that more appropriate treatment modalities can be offered to OSCC patients in Taiwan, Japan, and worldwide.

\section{Acknowledgements}

We thank Dr Homare Kawachi for his help with collecting OSCC samples and supplying the clinical data for the preparation of this manuscript.

\section{References}

1. Sudbo J: Novel management of oral cancer: a paradigm of predictive oncology. Clin Med Res 2: 233-242, 2004.

2. Macfarlane GJ, Zheng T, Marshall JR, Boyle P, et al: Alcohol, tobacco, diet and the risk of oral cancer: a pooled analysis of three case-control studies. Oral Oncol 31: 181-187, 1995.

3. Mashberg A, Boffetta P, Winkelman R and Garfinkel L: Tobacco smoking, alcohol drinking, and cancer of the oral cavity and oropharynx among U.S. veterans. Cancer 72: 1369-1375, 1993.

4. Warnakulasuriya S, Sutherland G and Scully C: Tobacco, oral cancer, and treatment of dependence. Oral Oncol 41: 244-260, 2005.

5. Ries LAG, Harkins D, Krapcho M, et al: Cancer Statistics Review 1975-2003. National Cancer Institute, 2006.

6. Genden EM, Ferlito A, Bradley PJ, Rinaldo A and Scully C: Neck disease and distant metastases. Oral Oncol 39: 207-212, 2003.

7. Sabichi AL, Demierre MF, Hawk ET, Lerman CE and Lippman SM: Frontiers in cancer prevention research. Cancer Res 63: 5649-5655, 2003.

8. Spafford MF, Koch WM, Reed AL, et al: Detection of head and neck squamous cell carcinoma among exfoliated oral mucosal cells by microsatellite analysis. Clin Cancer Res 7: 607-612, 2001.

9. Fearon ER and Vogelstein B: A genetic model for colorectal tumorigenesis. Cell 61: 759-767, 1990.

10. Marshall CJ: Tumor suppressor genes. Cell 64: 313-326, 1991.

11. Garnis C, Baldwin C, Zhang L, Rosin MP and Lam WL: Use of complete coverage array comparative genomic hybridization to define copy number alterations on chromosome $3 p$ in oral squamous cell carcinomas. Cancer Res 63: 8582-8585, 2003.

12. Bjartell A, Johansson R, Björk T, et al: Immunohistochemical detection of cysteine-rich secretory protein 3 in tissue and in serum from men with cancer or benign enlargement of the prostate gland. Prostate 66: 591-603, 2006.

13. Horne AW, Duncan WC, King AE, et al: Endometrial cysteinerich secretory protein 3 is inhibited by human chorionic gonadotrophin, and is increased in the decidua of tubal ectopic pregnancy. Mol Hum Reprod 15: 287-294, 2009.

14. Udby L, Cowland JB, Johnsen AH, Sørensen OE, Borregaard N and Kjeldsen L: An ELISA for SGP28/CRISP-3, a cysteine-rich secretory protein in human neutrophils, plasma, and exocrine secretions. J Immunol Methods 263: 43-55, 2002.

15. Ye H, Yu T, Temam S, et al: Transcriptomic dissection of tongue squamous cell carcinoma. BMC Genomics 6: 9-69, 2008. 
16. Kato H, Uzawa K, Onda T, et al: Down-regulation of 1Dmyo-inositol 1,4,5-trisphosphate 3-kinase A protein expression in oral squamous cell carcinoma. Int J Oncol 28: 873-881, 2006.

17. UICC: TNM classification of malignant tumours. Fifth edition: 20-24, 1997.

18. Bolstad BM, Irizarry RA, Astrand M and Speed TP: A comparison of normalization methods for high density oligonucleotide array data based on variance and bias. Bioinformatics 19: 185-193, 2003

19. Irizarry RA, Hobbs B, Collin F, et al: Exploration, normalization, and summaries of high density oligonucleotide array probe level data. Biostatistics 4: 249-264, 2003.

20. Kuroiwa T, Yamamoto N, Onda T and Shibahara T: Expression of the FAM5C in tongue squamous cell carcinoma. Oncol Rep 22: 1005-1011, 2009.

21. Xu C, Liu Y, Wang P, et al: Integrative analysis of DNA copy number and gene expression in metastatic oral squamous cell carcinoma identifies genes associated with poor survival. Mol Cancer 9: 143-148, 2010.

22. La Vecchia C: Epidemiology and prevention of oral cancer. Oral Oncol 33: 302-312, 1997.

23. Martin CL, Reshmi SC, Ried T, et al: Chromosomal imbalances in oral squamous cell carcinoma: examination of 31 cell lines and review of the literature. Oral Oncol 44: 369-382, 2008.

24. Cohen DJ, Busso D, Da Ros V, et al: Participation of cysteinerich secretory proteins (CRISP) in mammalian sperm-egg interaction. Int J Dev Biol 52: 737-742, 2008.

25. Gibbs GM, Scanlon MJ, Swarbrick J, et al: The cysteine-rich secretory protein domain of Tpx-1 is related to ion channe toxins and regulates ryanodine receptor $\mathrm{Ca}^{2+}$ signaling. $\mathrm{J}$ Bio Chem 281: 4156-4163, 2006.
26. Gibbs GM, Bianco DM, Jamsai D, et al: Cysteine-rich secretory protein 2 binds to mitogenactivated protein kinase 11 in mouse sperm. Biol Reprod 77: 108-114, 2007.

27. Gibbs GM and O'Bryan MK: Cysteine rich secretory proteins in reproduction and venom. Soc Reprod Fertil 65: 261-267, 2007.

28. Udby L, Lundwall $\AA$, Johnsen AH, et al: $\beta$-microseminoprotein binds CRISP-3 in human seminal plasma. Biochem. Biophys Res Commun 333: 555-561, 2005.

29. Guo M, Ren J, Brock MV, Herman JG, and Carraway HE Promoter methylation of HIN-1 in the progression to esophageal squamous cancer. Epigenetics 3: 336-341, 2008.

30. Castro M, Grau L, Puerta P, et al: Multiplexed methylation profiles of tumor suppressor genes and clinical outcome in lung cancer. J Transl Med 8: 86-96, 2010.

31. Bai Z, Ye Y, Liang B, et al: Proteomics-based identification of a group of apoptosis-related proteins and biomarkers in gastric cancer. Int J Oncol 38: 375-383, 2011.

32. Maresh EL, Mah V, Alavi M, et al: Differential expression of anterior gradient gene AGR2 in prostate cancer. BMC Cancer 13: 680-687, 2010.

33. Brinckerhoff CE and Matrisian LM: Matrix metalloproteinases: a tail of a frog that became a prince. Nat Rev Mol Cell Biol 3: 207-214, 2002.

34. Egeblad M and Werb Z: New functions for the matrix metalloproteinases in cancer progression. Nat Rev Cancer 2: 161-174, 2002.

35. Nagase $\mathrm{H}$ and Woessner JF Jr: Matrix metalloproteinases. J Biol Chem 274: 21491-21494, 1999.

36. Ala-aho R and Kahari VM: Collagenases in cancer. Biochimie 87: 273-286, 2005 